\title{
Naringenin regulates production of matrix metalloproteinases in the knee-joint and primary cultured articular chondrocytes and alleviates pain in rat osteoarthritis model
}

\author{
C.C. Wang ${ }^{1}$, L. Guo ${ }^{1}$, F.D. Tian $^{1}$, N. An ${ }^{1}$, L. Luo ${ }^{2}$, R.H. Hao ${ }^{1}$, B. Wang ${ }^{1}$ and Z.H. Zhou ${ }^{1}$ \\ ${ }^{1}$ Department II of Orthopedics, Affiliated Zhongshan Hospital of Dalian University, Dalian, Liaoning, China \\ ${ }^{2}$ Department of Respiratory Medicine, Affiliated Zhongshan Hospital of Dalian University, Dalian, Liaoning, China
}

\begin{abstract}
Inflammation of cartilage is a primary symptom for knee-joint osteoarthritis. Matrix metalloproteinases (MMPs) are known to play an important role in the articular cartilage destruction related to osteoarthritis. Naringenin is a plant-derived flavonoid known for its anti-inflammatory properties. We studied the effect of naringenin on the transcriptional expression, secretion and enzymatic activity of MMP-3 in vivo in the murine monosodium iodoacetate (MIA) osteoarthritis model. The assessment of pain behavior was also performed in the MIA rats. The destruction of knee-joint tissues was analyzed microscopically. Moreover, the effect of naringenin was also studied in vitro in IL-1 $\beta$ activated articular chondrocytes. The transcriptional expression of MMP-3, MMP-1, MMP-13, thrombospondin motifs (ADAMTS-4) and ADAMTS-5 was also studied in primary cultured chondrocytes of rats. Naringenin caused significant reduction in pain behavior and showed marked improvement in the tissue morphology of MIA rats. Moreover, a significant inhibition of MMP-3 expression in MIA rats was observed upon treatment with naringenin. In the in vitro tests, naringenin caused a significant reduction in the transcriptional expression, secretion and enzymatic activity of the studied degradative enzymes. The NF-kB pathway was also found to be inhibited upon treatment with naringenin in vitro. Overall, the study suggests that naringenin alleviated pain and regulated the production of matrix-metalloproteinases via regulation of NF-kB pathway. Thus, naringenin could be a potent therapeutic option for the treatment of osteoarthritis.
\end{abstract}

Key words: Metalloproteinase; Osteoarthritis; Naringenin; Chondrocyte; Knee-joint; Rat MIA model

\section{Introduction}

Millions of people, mostly elderly, are affected by osteoarthritis (OA) all over the world. The symptoms of osteoarthritis include inflammation of synovial joints, osteophyte formation and articular cartilage degeneration. Although, clear understanding of the causes of osteoarthritis remains elusive, several causes such as physiologic synthesis disruption and articular cartilage degradation during osteoarthritis progression have been postulated $(1,2)$.

Matrix metalloproteinases (MMPs) play a central role in articular cartilage destruction in patients with osteoarthritis $(3,4)$. MMPs have been classified into gelatinases (MMP-2, MMP-9), collagenases (MMP-1, MMP-8, MMP-13) and stromelysins (MMP-3, MMP-7, MMP-10, MMP-11) $(5,6)$. Of these MMPs, MMP-3 is most well-known for its role in degradation of proteoglycans and also activation of procollagenase $(7,8)$. Several plant-derived products are known for their anti-arthritic properties (9). Therefore, studies should clinically test any natural remedy that could have an effect on the expression and activity of MMP-3 and other matrix metalloproteinases.

The present therapeutic regimen of osteoarthritis primarily involves pain management by using non-steroidal anti-inflammatory drugs (NSAIDs) (10). Although the NSAID therapy of osteoarthritis is well established, chronic NSAID use may lead to several side effects including gastrointestinal damage $(11,12)$. Therefore, use of natural therapeutic agents along with traditional NSAIDs has been proposed (13).

Several flavonoids are known for their role in alleviation of osteoarthritis $(14,15)$. Naringenin is a plant-derived flavanone found in tomato, grapefruit and citrus fruits (16). Naringenin is known for its antioxidant and anti-inflammatory properties $(17,18)$. Naringenin has also been shown to have a role in modulation of MMP-2 and MMP-9 via inhibition of

Correspondence: L. Guo: <guolin1391@hotmail.com> 
NF-kB pathway (19). However, the role of naringenin in modulation of MMP-3 in osteoarthritis has not yet been explored. In this study, we explored the chondro-protective activity of naringenin in a rat OA model. Specifically, we identified the effect of naringenin on the alleviation of pain associated with osteoarthritis in monosodium iodoacetate (MIA) murine OA model. The effects of naringenin on in vivo MMP-3 secretion and on IL-1 $\beta$-induced gene expression of several degradative enzymes including MMPs in vitro were studied. We also attempted to identify the possible mechanism of regulation of degradative enzyme levels upon induction with IL-1 $\beta$ in vitro.

\section{Material and Methods}

Male Wistar rats (220-240 g; n=45) were used to determine the effect of naringenin on the alleviation of OA. The animals were kept under a 12/12 hour light and dark circadian cycle and under controlled conditions of temperature and humidity. The animals were fed standard rat diet and water ad libitum. All animals' up-keeping procedures were carried out according to the guidelines of World Medical Association Animal Ethics Guidelines (declaration of Helsinki).

\section{Induction of OA in rats and experimental groups}

The animals were anesthetized with isoflurane, and then 2 mg MIA (Sigma-Aldrich, USA) was injected in the intra-articular space of the right knee using a $26.5 \mathrm{G}$ needle in $30 \mu \mathrm{L}$ volume. The control group was injected with phosphate buffered saline (PBS). The animals were assigned to three treatment groups at random with 15 animals per group as follows: 1) control group that was administered with PBS; 2) $20 \mathrm{mg} / \mathrm{kg}$ naringenin; and 3) $40 \mathrm{mg} / \mathrm{kg}$ naringenin. Naringenin was obtained from Sigma-Aldrich (USA) and dissolved in 5\% dimethyl sulfoxide (DMSO) and administered orally by gavage in $300 \mu \mathrm{L}$ volume doses daily. Naringenin treatment commenced two days after induction of OA by MIA.

\section{Assessment of pain behavior in MIA rats}

A dynamic planter esthesiometer (Ugo Basile, Italy) was used for the nociceptive testing of animals. Testing was performed prior to MIA induction and subsequently each day until the end of the experiment ( 2 weeks). Before testing, the animals were rested for $30 \mathrm{~min}$ in a controlled environment chamber. The touch-stimulator of the instrument was aligned under the animal. The angled mirror was used to orient the stimulating micro-filament below the hind-paw planter surface. Upon activation, the $0.5 \mathrm{~mm}$ microfilament progressed to touch the proximal region of the metatarsal area. Starting below detection threshold, a gradually increasing stimulating force was applied by the filament until retraction of the paw occurred indicating pain stimulus. The pain stimulating force was automatically recorded. A maximum force of $500 \mathrm{~N}$ and a ramp speed of $20 \mathrm{~s}$ were employed in all the tests.

\section{Effect of naringenin on in vivo secretion of MMP-3}

The animals were euthanized at the end of treatment using the $\mathrm{CO}_{2}$ overdose method. Isolation of the rat knee articular cartilage was performed. For isolation, the tissue was homogenized and protein concentration was estimated using Bradford's method. Fifteen micrograms of the estimated protein was loaded per well on $12 \%$ sodium dodecyl sulfate polyacrylamide gel electrophoresis (SDSPAGE) gel. The proteins were electrophoretically transferred on a polyvinylidene difluoride (PVDF)-membrane after completion of the gel run. Blocking of the membranes was performed for $1.5 \mathrm{~h}$ in Tris-buffered saline, which contained $0.2 \%$ Tween 20 (TBST) and $2 \%$ non-fat dry milk. MMP-3 antibodies were obtained from Cell Signaling Technology, Inc. (USA). The binding of antibodies onto membranes was performed by incubating it with primary antibody solution in TBST and $2 \%$ non-fat dry milk for $8 \mathrm{~h}$ at $4^{\circ} \mathrm{C}$. The membrane was washed thrice with TBST. Finally, the membranes incubated for $1 \mathrm{~h}$ with horseradish peroxidase (HRP) conjugated secondary antibodies and developed using an ECL detection system. The band density on the membranes was scanned and then analyzed using the Image $(\mathrm{NIH}$, USA) software program.

\section{Histopathological analysis of rat knee}

The knee tissues were isolated from sacrificed animals and fixed in $12 \%$ neutral buffered formaldehyde solution for $72 \mathrm{~h}$ at $4^{\circ} \mathrm{C}$. The decalcification of tissues was performed in formic acid for 6 days. Subsequently, the tissue was dehydrated in increasing concentration of ethanol. The dehydrated tissues were cleared with xylene and fixed in paraffin. Sections of five to seven microns were cut using microtome and stained with hematoxylin and eosin. The morphological differences between the sections of different treatment groups were scored following the modified Mankin scoring system. For scoring, morphological characteristics were analyzed as follows: N: normal morphology, + : mild damage, ++ : severe damage. The scoring was performed twice by two independent researchers who were blind to the treatment experiment.

\section{Primary culture of articular chondrocytes and treatment with naringenin}

Articular tissues of the animals were isolated from femoral condyle and tibial plateau. The isolated tissues were washed with PBS, ground to small pieces and digested with $0.3 \%$ solution of collagenase for $3 \mathrm{~h}$ at $37^{\circ} \mathrm{C}$. The digested homogenate was centrifuged at $10,000 \mathrm{~g}$ for $1 \mathrm{~min}$ at $4^{\circ} \mathrm{C}$ and the cell pellet was collected. The individual cells were transplanted to $100 \mathrm{~mm}$ culture plates with a seeding density of $10^{5}$ cells $/ \mathrm{cm}^{2}$ in $10 \mathrm{~mL}$ of DMEM, $12 \%$ fetal bovine serum, penicillin (100 units $/ \mathrm{mL})$ and streptomycin $(100 \mu \mathrm{g} / \mathrm{mL})$. The cells were cultured under $5 \% \mathrm{CO}_{2} / 95 \%$ air at $37^{\circ} \mathrm{C}$. The culture medium was replaced daily.

The chondrocytes were seeded on 6-well culture plates at a culture density of $10^{5}$ cells $/ \mathrm{cm}^{2}$. After 2 days of culture, the cells were incubated with 20 and $40 \mu \mathrm{M}$ of naringenin for 


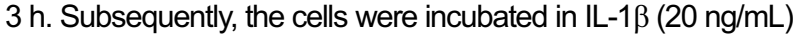
for $24 \mathrm{~h}$ with a negative control of absence of IL-1 $\beta$. Naringenin solution was prepared in dimethyl sulfoxide (DMSO) and PBS. The final concentration of DMSO was kept at $0.75 \%$ and $\mathrm{pH} 7.0-7.5$. No effect of culture medium was found on the various parameters of MMP-3 activity.

\section{Isolation of total RNA and qRT-PCR}

Total RNA was isolated from chondrocytes using the RNeasy Mini Kit (Qiagen, USA). Subsequently, qRT-PCR was performed using One-Step qRT-PCR Kit (Qiagen) following the manufacture's protocol. The designing of qRT-PCR primers was done using Primer-BLAST (21). The melting temperature $(\mathrm{Tm})$ of primers was taken between $57^{\circ} \mathrm{C}$ and $63^{\circ} \mathrm{C}$. The size of amplification product was taken between 90 and $120 \mathrm{bp}$ along with difference in $\mathrm{Tm}$ of $3^{\circ} \mathrm{C}$ (max). We took three technical replicates for every biological replicate. The quality of RNA was assessed by a QIAxpert ${ }^{\mathrm{TM}}$ microfluidic UV/VIS spectrophotometer (Qiagen). The PCR master mix included $5 \mu \mathrm{L}$ DyNAmo Flash SYBR Green (Thermo) (2X), $1.5 \mu \mathrm{L}$ cDNA, $1 \mu \mathrm{L}(5 \mathrm{pm} / \mu \mathrm{L})$ each primer. The following cycling conditions were used for qRT-PCR: denaturation at $95^{\circ} \mathrm{C}$ for $10 \mathrm{~min}, 40$ denaturation cycles at $95^{\circ} \mathrm{C}$ for $22 \mathrm{~s}$, annealing with extension at $60^{\circ} \mathrm{C}$ for $60 \mathrm{~s}$. The qRT-PCR amplification was performed in ABI 7500 system (Applied Biosystems, USA). The threshold cycle value $(\mathrm{Ct})$ for the genes were quantified and normalized by $\mathrm{Ct}$ value for glyceraldehyde-3-phosphate dehydrogenase (GADPH) expression. The following primers were used for qRT-PCR: MMP-3 (5'-TTTGGCCGTCTCTTC CATCC-3', 5'-GGAGGCCCAGAGTGTGAATG-3'), MMP13 (5'-GGACTCACTGTTGGTCCCTG-3'; 5'-GGATTCC CGCAAGAGTCACA-3'), MMP-1 (5'-CCGGCAGAATGTG GAAACAG-3', 5'-GCTGCATTTGCCTCAGCTTT-3'), ADAM TS-4 (5'-CATCCTACGCCGGAAGAGTC-3', 5'-AAGCGAA GCGCTTGTTTCTG-3'), and ADAMTS-5 (5'-CCCAAATAC GCAGGTGTCCT-3'; 5'-ACACACGGAGTTGCTGTAGG-3'). GAPDH (5'-TGTGAACGGATTTGGCCGTA-3'; 5'-TGAACTT GCCGTGGGTAGAG- $3^{\prime}$ ) was used as an internal control.

\section{Assay for assessment of cytotoxicity of naringenin}

The chondrocytes were seeded on a 96-well microtiter plate at a density of $2 \times 10^{5}$ cells $/ \mathrm{mL}$ and were allowed to attach onto the surface for $24 \mathrm{~h}$, which allowed optimal treatment with naringenin during the log-phase of cell growth. Naringenin solution was prepared in DMSO in a graded set of solutions ranging from 10 to $100 \mu \mathrm{M}$. Treatment with naringenin was performed in DMEM and $10 \%$ FBS. The cells were incubated for $72 \mathrm{~h}$ and the proliferation of cells was estimated by the sulforhodamine-B (SRB) assay (20).

\section{Estimation of MMP-3 and phosphorylated forms of NF- $\kappa B$ and $I \kappa B-\alpha$ levels in the articular chondrocytes culture supernatants}

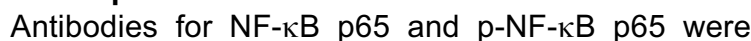
obtained from Santa Cruz Biotechnology, Inc. (USA) and
anti-MMP-3, anti- $\mid \kappa B-\alpha$ and anti-p- $\mid \kappa B-\alpha$ antibodies were obtained from Cell Signaling Technology, Inc. The concentrations of the proteins in the culture supernatants were estimated using Bradford's method as mentioned above. Fifteen micrograms of the estimated protein was loaded per well on $12 \%$ SDS-PAGE gel. Western blot densitometry was performed as described above.

\section{Estimation of MMP-3 proteolytic activity}

Chondrocyte culture supernatants were pretreated for $3 \mathrm{~h}$ with naringenin. Subsequently, IL-1 $\beta(20 \mathrm{ng} / \mathrm{mL})$ was used for stimulation of these culture supernatants in medium containing DMEM and FBS (0.5\%). Protein concentrations were estimated using Bradford's method and $15 \mu \mathrm{g}$ of the estimated protein was loaded per well on $12 \%$ SDS-PAGE gel with casein (0.1\%), $10 \mathrm{mM}$ Tris- $\mathrm{HCl}$ at $\mathrm{pH}$ 8.0. The gels were washed with $10 \mathrm{mM}$ Tris- $\mathrm{HCl}$ (pH 8.0) containing 2\% Triton X-100 after electrophoresis. After washing, the gels were incubated in $100 \mathrm{mM}$ Tris$\mathrm{HCl}, \mathrm{pH} 8.0$, with $2 \%$ Triton-X100, $0.1 \mathrm{M} \mathrm{NaCl}, 4 \mathrm{mM}$ $\mathrm{CaCl}_{2}$ for $48 \mathrm{~h}$ at $37^{\circ} \mathrm{C}$. Coomassie blue staining of the gels was performed and the band density on the membranes was scanned and then analyzed using the ImageJ (NIH, USA) software program.

\section{Statistical analysis}

The mean values of the individual experimental groups were calculated as percentage of the control group. The individual averages are reported as average \pm SEM. The data for assessment of pain behavior are reported as average \pm SEM. The variance between the experimental groups was estimated using one-way ANOVA with Bonferonni's multiple corrections for post hoc analysis. $\mathrm{P} \leqslant 0.05$ was considered to be significant. All statistical analyses were performed in Statistica v8.0 (StatSoft, USA).

\section{Results}

\section{Effect of naringenin treatment on pain generation in OA rats}

In the pain assessment test, parameters such as paw withdrawal latency (PWL) and paw withdrawal threshold (PWT) showed a significant prolongment upon treatment with naringenin when compared with the MIA group (Figure 1). These results showed that pain alleviation began from the fourth day of the treatment. It was also found that at a dose of $40 \mathrm{mg} / \mathrm{kg}$ the effect of naringenin was more pronounced and significant compared to the $20 \mathrm{mg} / \mathrm{kg}$ naringenin group.

\section{Effect of naringenin on in vivo secretion of MMP-3}

To determine the effect of naringenin on in vivo expression of MMP-3 in the articular cartilage tissues, western blot densitometry for MMP-3 was performed. In the MIA rats, there was a significantly greater MMP-3 


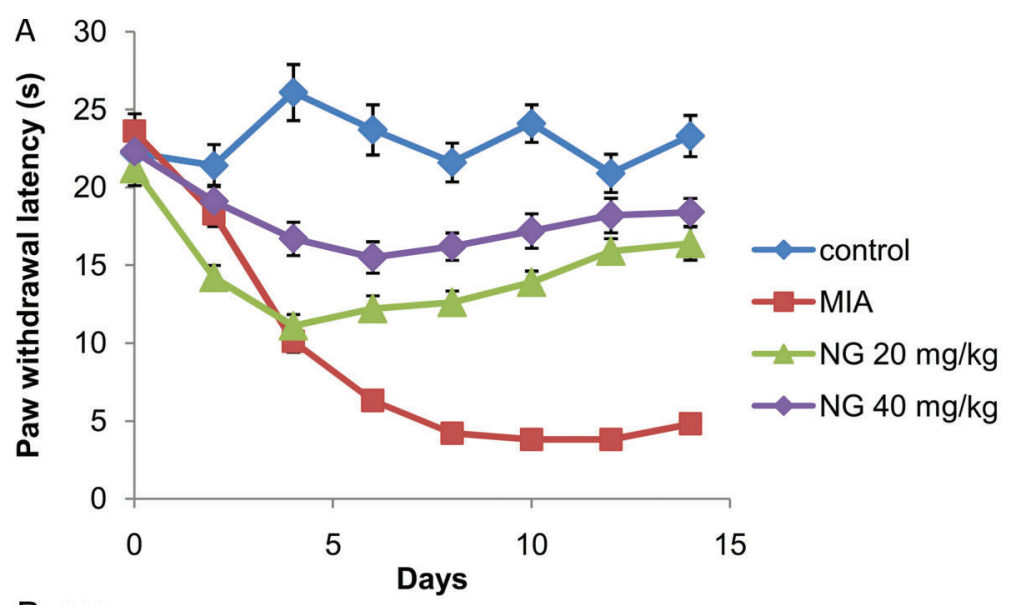

Figure 1. Effect of naringenin (NG) on mechanical hyperalgesia in a model of monosodium iodoacetate (MIA)-induced osteoarthritis in rats. Two milligrams of MIA was injected, and 2 days later, NG was administered orally for 2 weeks at 20 and $40 \mathrm{mg} / \mathrm{kg}$. A, Paw withdrawal latency (s). B, Paw withdrawal threshold $(\mathrm{N})$. Data are reported as means \pm SEM. ${ }^{*} P \leqslant 0.05$ and ${ }^{* *} P \leqslant 0.01$ compared to MIA group; ${ }^{\#} \mathrm{P} \leqslant 0.05$ and ${ }^{\# \#} \mathrm{P} \leqslant 0.01$ compared to MIA group (ANOVA with Bonferonni's post hoc multiple corrections).

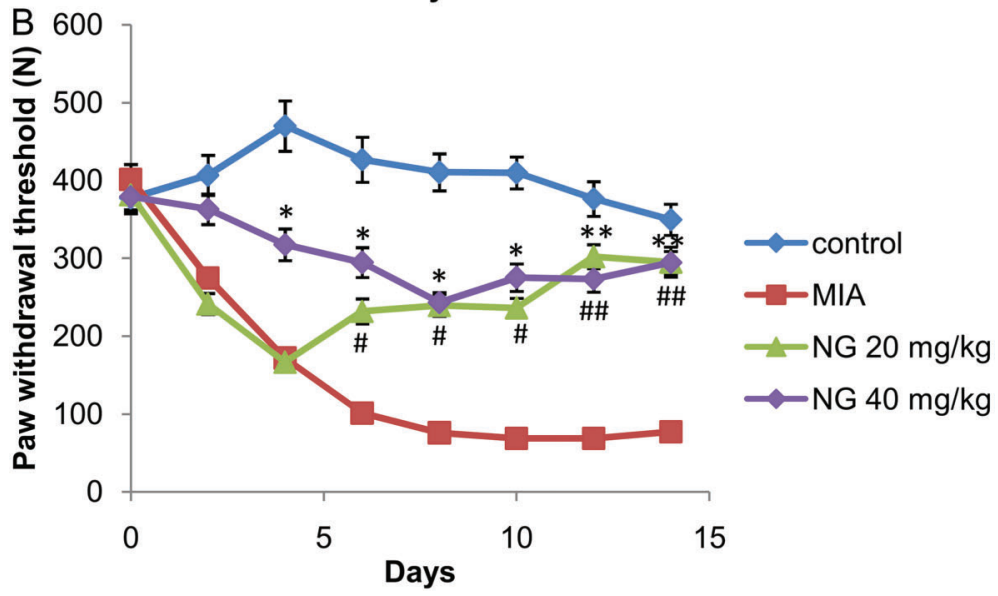

activity compared to control animals (Figure 2). Upon treatment with naringenin, a significant decrease in MMP-3 activity was observed. The greatest decrease was observed at $40 \mathrm{mg} / \mathrm{kg}$ naringenin.

\section{Effect of naringenin on knee histopathology}

The effect of oral administration of naringenin on MIA induced-osteoarthritis is shown in Table 1. The induction of OA caused mild to severe chondrocyte death, degeneration of muscle tissue, erosion of cartilage and fibrillation (Figure 3) compared to the control group. The administration of $20 \mathrm{mg} / \mathrm{kg}$ naringenin caused sub-maximal effect on those parameters. However, administration of $40 \mathrm{mg} / \mathrm{kg}$ naringenin showed marked improvement in the knee tissue morphology. A significant attenuation of chondrocyte death, muscle degradation, cartilage erosion and fibrillation was observed in knee tissues at $40 \mathrm{mg} / \mathrm{kg}$.

Effect of naringenin on the transcriptional expression of MMP-3, MMP-1, MMP-13, ADAMTS-4, and ADAMTS-5

We determined the effect of naringenin on the expression of MMPs and thrombospondin motifs in IL-1 $\beta$ stimu- lated chondrocyte cells. The expression of MMP-3, MMP-1 and MMP-13 showed a significant reduction in naringenintreated cells. Similarly, the expression of ADAMTS-4, and ADAMTS-5 was also significantly reduced upon treatment with naringenin of IL-1 $\beta$-treated chondrocytes. In all cases, gene expression of MMP-1, MMP-3, MMP-13, ADAMTS-4 and ADAMTS-5 showed a significant decrease with naringenin treatment (Figure 4A). In this analysis, the effect of naringenin was also found to be dose-dependent.

\section{Effect of naringenin on chondrocyte proliferation}

The cytotoxicity of naringenin on rat articular chondrocytes was estimated from 10 to $100 \mu \mathrm{M}$ concentration. No significant difference in cytotoxicity was found for naringenin compared to control (Figure 4B). Moreover, at all concentrations, the numbers for cell proliferation was almost the same without a dose-dependent trend.

\section{Effect of naringenin on IL-1 $\beta$-induced secretion of MMP-3 from rat articular chondrocytes}

A significant decrease in gene expression of MMP-3 upon treatment with naringenin was found. Therefore, western blot 

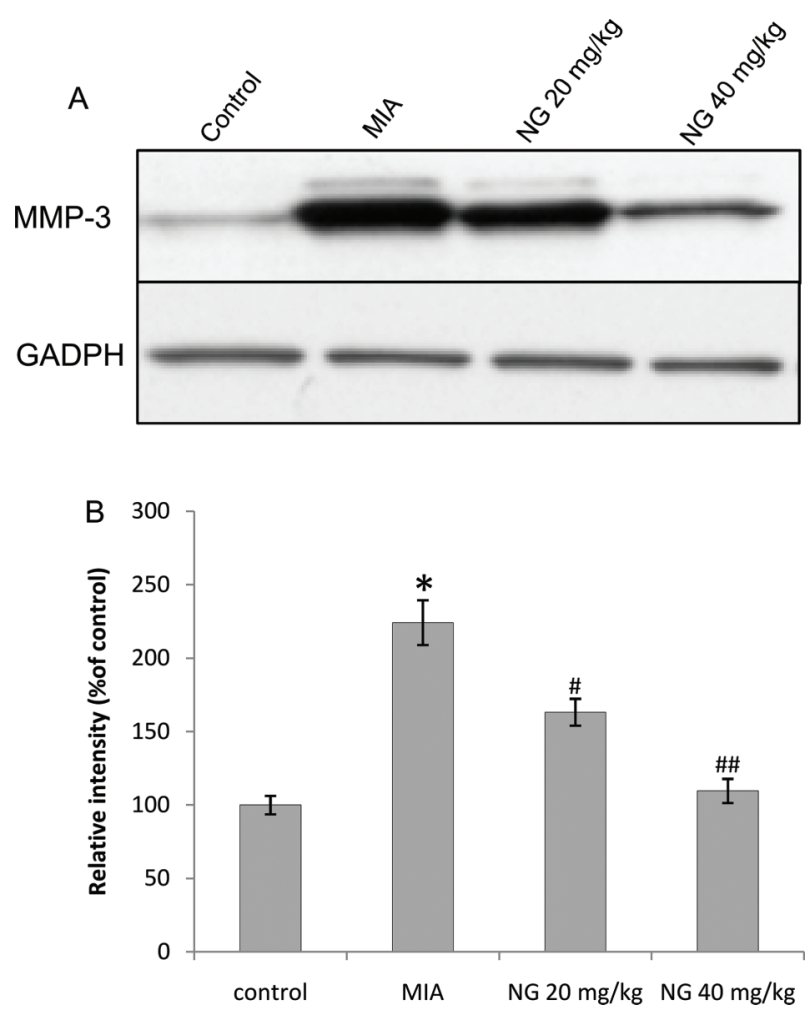

Figure 2. Effect of naringenin (NG) on the production of MMP-3 in vivo. Naringenin was administered orally for 2 weeks at 20 and 40 $\mathrm{mg} / \mathrm{kg}$, beginning 2 days post-induction of osteoarthritis by $2 \mathrm{mg}$ of monosodium iodoacetate (MIA). Total protein was isolated from rat knee articular cartilage. MMP-3 concentration was estimated using western blot densitometry. A, Western blot of MMP-3 and $\mathrm{GADPH} ; B$, relative intensity of bands. Data are reported as means \pm SEM. ${ }^{*} \mathrm{P} \leqslant 0.05$, compared to negative control (PBS); ${ }^{\#} \mathrm{P} \leqslant 0.05$ and ${ }^{\# \#} \mathrm{P} \leqslant 0.01$, compared to MIA-treated group (ANOVA with Bonferonni's post hoc multiple corrections).

Table 1. Effect of naringenin on morphology of knee tissue of murine monosodium iodoacetate-induced (MIA) osteoarthritis in rats.

\begin{tabular}{lcccc}
\hline & Control & MIA & $20 \mathrm{mg} / \mathrm{kg}$ & $40 \mathrm{mg} / \mathrm{kg}$ \\
\hline Chondrocyte death & $\mathrm{N}$ & + & ++ & $\mathrm{N}$ \\
Muscle degeneration & $\mathrm{N}$ & ++ & $\mathrm{N}$ & + \\
Erosion of cartilage & $\mathrm{N}$ & ++ & + & + \\
Fibrillation & $\mathrm{N}$ & ++ & + & $\mathrm{N}$ \\
\hline
\end{tabular}

A modified Mankin scoring analysis was carried out. Naringenin was administered orally for 2 weeks at 20 and $40 \mathrm{mg} / \mathrm{kg}$ beginning 2 days post-induction of osteoarthritis by $2 \mathrm{mg}$ MIA. Knee tissue was collected at the end of treatment ( 2 weeks). $\mathrm{N}$ : normal morphology, + : mild damage, ++ : severe damage.

analysis was performed to identify if this transcriptional change was also observable in translation. There was a large increase in MMP-3 production upon IL-1 $\beta$ induction
(Figure 5). Treatment with naringenin caused a significant decrease in the protein concentration of MMP-3 in a dosedependent manner.

\section{Effect of naringenin on phosphorylated forms of NF- $\kappa \mathrm{B}$ and $\mathrm{I} \kappa \mathrm{B}-\alpha$}

The in vivo protein expression of NF- $\kappa \mathrm{B}$ and $1 \kappa \mathrm{B}-\alpha$ and their phosphorylated forms was estimated using western blot densitometry. As illustrated in Figure 6, OA induction with MIA resulted in 17- and 11 -fold increases $(P<0.01)$ in the phosphorylation of NF- $\mathrm{NB}$ p 65 and $\mathrm{I} \kappa \mathrm{B}-\alpha$, respectively, compared to the control group. However, a significant and dose-dependent decrease in the NF-кB p65 and $I_{\kappa} B-\alpha$ phosphorylation was observed upon treatment with naringenin. These results indicate that naringenin could inhibit the NF- $\mathrm{KB}$ activation due to $\mathrm{OA}$ induction.

\section{Effect of naringenin on proteolytic activity of MMP-3 in rat articular chondrocytes}

To determine the effect of naringenin on the enzymatic activity of MMP-3, we determined the caseinolytic activity of MMP-3 in the rat articular chondrocytes using casein zymography. We found a large and significant increase in the MMP-3 activity upon IL-1 $\beta$ induction (Figure 7 ). This induction showed a significant decrease upon pre-treatment with naringenin in a dose-dependent manner as was evident from the Coomassie blue stained SDS-PAGE gel.

\section{Discussion}

Osteoarthritis is a degenerative disease of articular chondrocytes and involves several inflammatory pathways and mediators such as IL-6, IL-1 $\beta$ and NO (22). MMPs are known to degrade the extracellular matrix components and elevated activity of MMPs has been associated with degradation of cartilage in OA patients. IL-1 $\beta$ is a proinflammatory cytokine, which causes up-regulation of MMPs in the articular chondrocytes, contributing to destruction of cartilages (23-25). Naringenin is a plant-derived flavonoid known to possess anti-inflammatory properties. However, no study has been performed on the identification of its effect on alleviation of OA. In this study, for the first time, we report the effect of naringenin on the expression, production and protein degradation activity of MMPs in vivo in MIA rat model as well as in vitro in IL-1 $\beta$-induced MMP production in cultured chondrocytes. We also report the alleviation of pain after treatment with naringenin in MIA rats.

The patho-physiology of pain related to OA is poorly understood. Cartilage is devoid of nerve endings and, thus, it is not the source of pain. OA-related pain arises from afferent nerve activation in the synovium, joint capsule, ligaments, periosteum etc. (26). Pain alleviation is an important goal in the treatment of OA. MIA-induced OA model is well characterized for joint pain (27), and mechanical hyperalgesia is exhibited by this model. The development of lesions in subchondral bone is similar to 

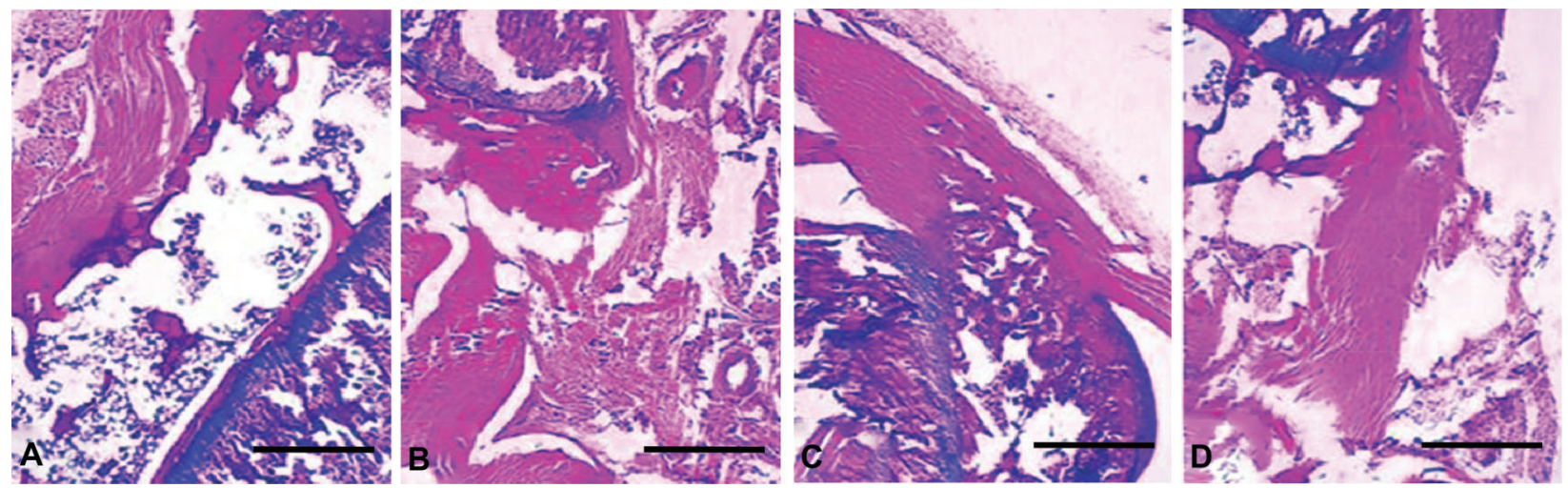

Figure 3. Effect of naringenin on histopathology of knee tissue of monosodium iodoacetate (MIA) rats. Naringenin was administered orally for 2 weeks at 20 and $40 \mathrm{mg} / \mathrm{kg}$ beginning 2 days post-induction of osteoarthritis by $2 \mathrm{mg}$ of MIA. The knee tissue was collected at the end of treatment (2 weeks). A, Negative control, $B$, MIA-treated, $C$, naringenin $20 \mathrm{mg} / \mathrm{kg}, D$, naringenin $40 \mathrm{mg} / \mathrm{kg}$. See Table $1 \mathrm{for}$ the results of Mankin scoring analysis. Magnification bar: $200 \mu \mathrm{M}$.

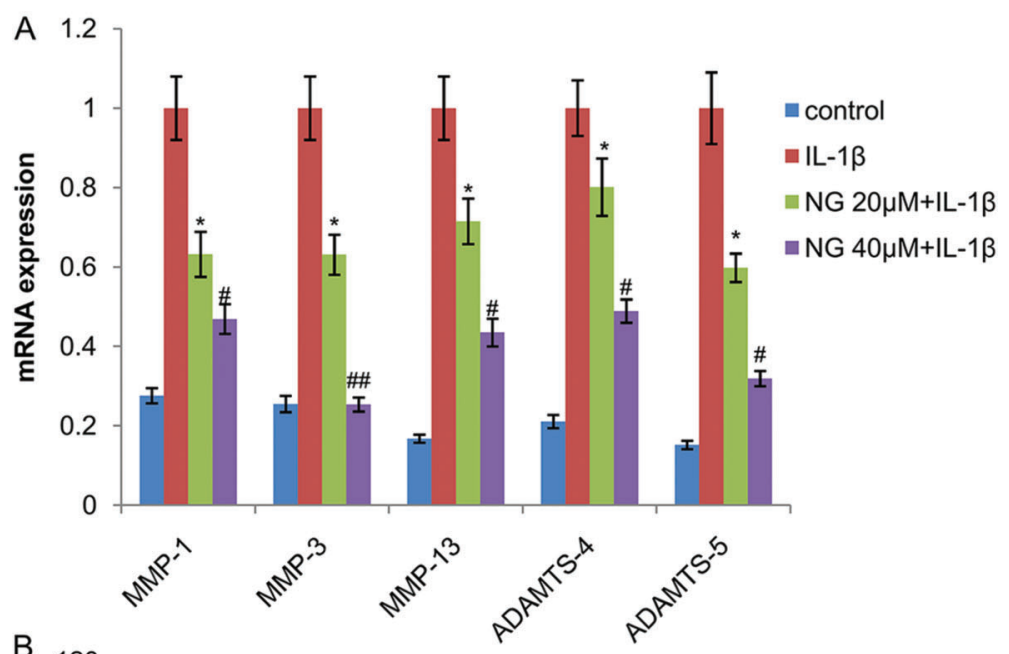

Figure 4. $A$, Effect of naringenin (NG) on gene expression of MMP-1, MMP-3, MMP-13, ADAMTS4 , and ADAMTS-5 in rat chondrocytes. The rat articular chondrocytes were cultured for 2 days and were incubated with 20 and $40 \mu \mathrm{M}$ of NG for $3 \mathrm{~h}$. Subsequently, the cells were incubated in IL-1 $\beta$ $(20 \mathrm{ng} / \mathrm{mL})$ for $24 \mathrm{~h}$ with a negative control of absence of IL-1 $\beta$. B. Effect of NG on proliferation of rat chondrocytes. Seeding of chondrocytes was performed $\left(2 \times 10^{5}\right.$ cells $\left./ \mathrm{mL}\right)$ for $24 \mathrm{~h}$ under varying concentrations of naringenin ranging from 10 to $100 \mu \mathrm{M}$. The proliferation of cells was estimated by sulforhodamine-B (SRB) assay. Data are reported as means $\pm S E M$. ${ }^{*} P \leqslant 0.05$, compared to IL- $1 \beta$; ${ }^{\#} \mathrm{P} \leqslant 0.05$ and ${ }^{\# \#} \mathrm{P} \leqslant 0.01$, compared to $\mathrm{IL}-1 \beta$ (ANOVA with Bonferonni's post hoc multiple corrections).

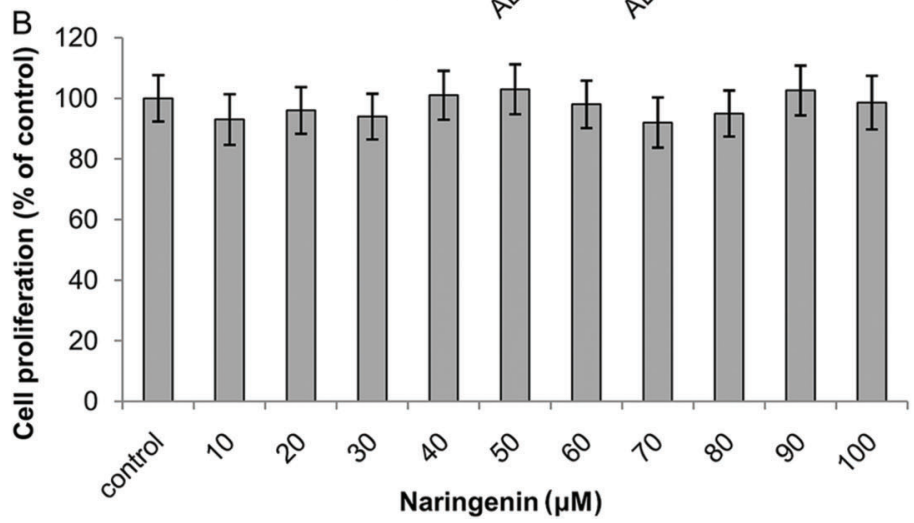

such lesions and pain in human osteoarthritis (28-30). Thus, it can be said that the attenuation of OA symptoms in the knee-joint tissues observed in the histopathological analysis was in accordance with the pain-reducing effect of naringenin.
In the in vivo experiments, naringenin was found to significantly reduce the MMP-3 secretion in the articular cartilage. These results indicated that naringenin treatment can alleviate the OA symptoms by inhibiting the MMP-3 mediated articular cartilage degeneration when administered 

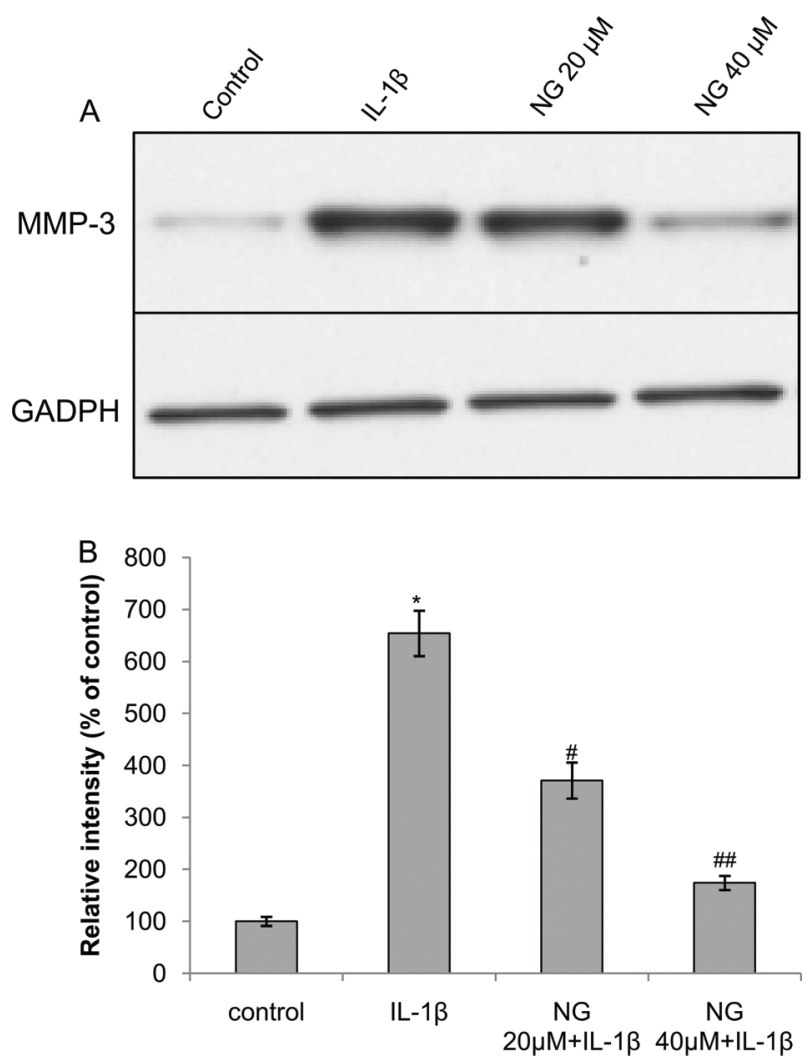

Figure 5. Effect of naringenin on MMP-3 secretion in rat chondrocytes. Primary cultured articular chondrocytes were pretreated with 20 and $40 \mu \mathrm{M}$ of naringenin. MMP-3 concentration was estimated in the total protein of articular chondrocytes using western blot densitometry. A, Western blot of MMP-3 and GADPH; $(B)$ relative intensity of bands. Data are reported as means \pm SEM. ${ }^{*} P \leqslant 0.05$, compared to negative control (PBS); ${ }^{\#} \mathrm{P} \leqslant 0.05$ and ${ }^{\#} \mathrm{P} \leqslant 0.01$, compared IL-1 $\beta$-treated culture (ANOVA with Bonferonni's post hoc multiple corrections).

orally. In addition, the in vivo MMP-3 levels were also significantly reduced in the cell cultures of articular chondrocytes induced by IL-1 $\beta$, as determined by the gene expression levels and western blot densitometry. Moreover, MMP-1 and MMP-13 also showed significant transcriptional downregulation when treated with naringenin. MMP-1 is generally found in the synovial fluid of OA patients (31-33). In addition to MMPs, the degradative enzymes ADAMTS-4 and ADAMTS-5 also showed significant down-regulation. ADMTS-4 and ADMTS-5 are known to cause destruction of matrix in OA $(34,35)$.

MMP-3 is also known to degrade proteoglycans (8). In order to estimate the effect of naringenin pre-treatment of the II-1 $\beta$-induced articular chondrocytes, casein zymography test was performed. A marked decrease in the proteolytic activity of MMP-3 was observed with naringenin
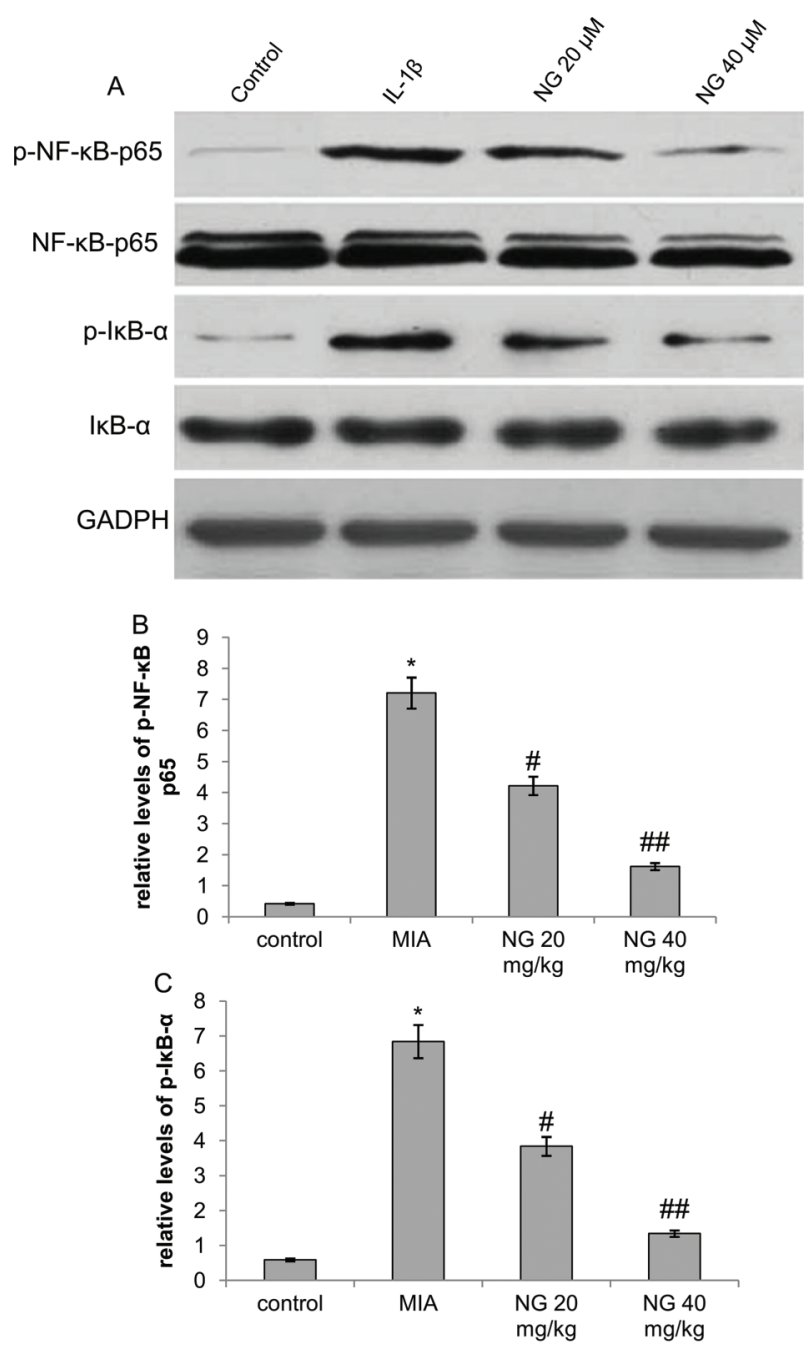

Figure 6. Effect of naringenin phosphorylated forms of NF-kB and I $\kappa \mathrm{B}-\alpha$. Primary cultured articular chondrocytes were pretreated with 20 and $40 \mu \mathrm{M}$ of naringenin. $A$, Western blot of NF-kB p65,

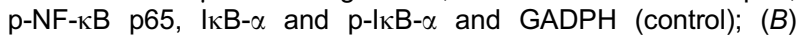
relative intensity of bands of $\mathrm{p}-\mathrm{NF}-\mathrm{\kappa} B \mathrm{p} 65 ;(C)$ relative intensity of bands of $p-I \kappa B-\alpha$. MIA: monosodium iodoacetate. Data are reported as means $\pm S E M$. ${ }^{*} P \leqslant 0.05$, compared to negative control (PBS); ${ }^{\#} \mathrm{P} \leqslant 0.05$ and ${ }^{\# \#} \mathrm{P} \leqslant 0.01$, compared IL-1 $\beta$ treated culture (ANOVA with Bonferonni's post hoc multiple corrections).

pre-treatment. These results indicated that, in addition to reduce expression and secretion of MMP-3, naringenin also reduced the proteolytic potential of MMP-3.

$N F-\kappa B$ is present in the cytoplasm in association with $I_{\kappa} B$. Abundance of the phosphorylated form of NF- $\kappa B$ and I $\kappa B$ has been reported in OA patients (36). Several studies have shown that NF- $\mathrm{KB}$ mediates the expression of MMP-1, MMP-3 and MMP-13 induced by cytokines such as IL-1 $\beta$ 


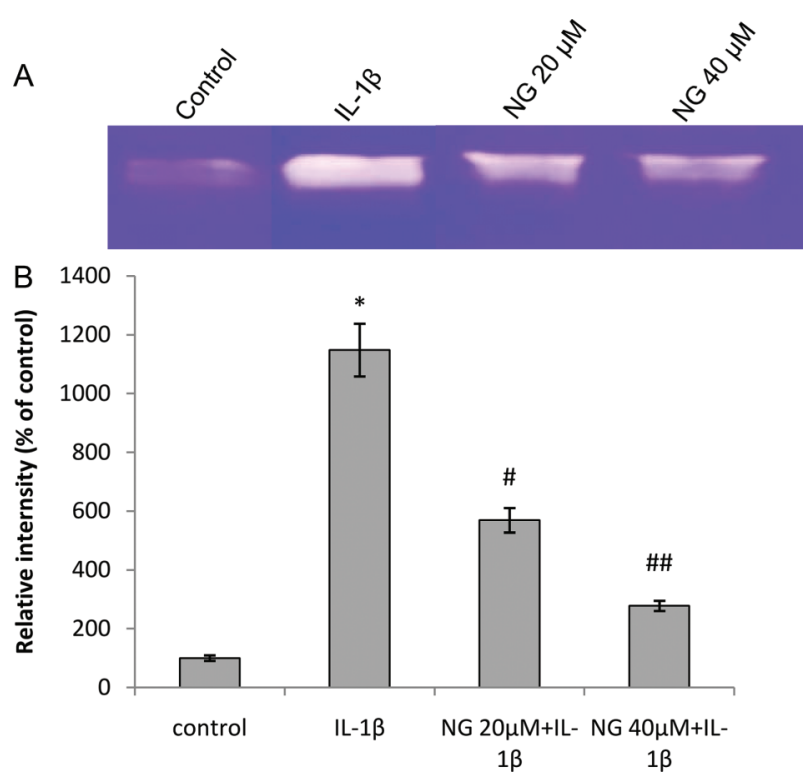

Figure 7. Effect of naringenin on caseinolytic activity of MMP-3 secretion in rat chondrocytes. Primary cultured articular chondrocytes were pretreated with 20 and $40 \mu \mathrm{M}$ of naringenin. MMP3 activity was estimated with casein zymography $(A)$ Coomassie blue-stained polyacrylamide gel of casein zymography; $(B)$ relative intensity of bands. Data are reported as means \pm SEM ${ }^{*} \mathrm{P} \leqslant 0.05$, compared to negative control (PBS); ${ }^{*} \mathrm{P} \leqslant 0.05$ and ${ }^{\# \#} \mathrm{P} \leqslant 0.01$, compared IL-1 $\beta$ treated culture (ANOVA with Bonferonni's post hoc multiple corrections)

(37). In this study, we found an elevated expression of the phosphorylated form of NF- $\mathrm{KB}$ and $\mathrm{I} \mathrm{\kappa B}$ in vitro in culture

\section{References}

1. Aigner T, McKenna L. Molecular pathology and pathobiology of osteoarthritic cartilage. Cell Mol Life Sci 2002; 59: 5-18, doi: 10.1007/s00018-002-8400-3.

2. Mankin HJ. The response of articular cartilage to mechanical injury. J Bone Joint Surg Am 1982; 64: 460-466, doi: 10.2106/00004623-198264030-00022.

3. Dean DD, Martel-Pelletier J, Pelletier JP, Howell DS, Woessner JF Jr. Evidence for metalloproteinase and metalloproteinase inhibitor imbalance in human osteoarthritic cartilage. J Clin Invest 1989; 84: 678-685, doi: 10.1172/ $\mathrm{JCl} 114215$.

4. Kullich W, Fagerer N, Schwann H. Effect of the NSAID nimesulide on the radical scavenger glutathione S-transferase in patients with osteoarthritis of the knee. Curr Med Res Opin 2007; 23: 1981-1986, doi: 10.1185/030079907X 223486.

5. Birkedal-Hansen H, Moore WG, Bodden MK, Windsor LJ, Birkedal-Hansen B, DeCarlo A, et al. Matrix metalloproteinases: a review. Crit Rev Oral Biol Med 1993; 4: 197-250, doi: $10.1177 / 10454411930040020401$.

6. Burrage PS, Mix KS, Brinckerhoff CE. Matrix metalloproteinases: role in arthritis. Front Biosci 2006; 11: 529-543, doi: $10.2741 / 1817$. supernatants after induction with IL-1 $\beta$. This expression was significantly reduced upon treatment with naringenin. Several studies have reported the inhibition of NF-KB pathway by flavonoids including naringenin $(38,39)$. Therefore, our results indicate an involvement of NF-kB pathway in the up-regulation of MMPs upon induction of OA. Furthermore, naringenin may have caused inhibition of the NF-kB pathway causing down-regulation of MMPs.

The present study showed the chondro-protective effect of naringenin on the expression and secretion of several degradative enzymes, particularly MMP-3. The enzymatic activity of MMP-3 was also found to be markedly decreased due to naringenin treatment, both in vivo and in vitro. However, naringenin showed no significant cytotoxicity on articular chondrocytes culture in vitro. Our results also indicate that the down-regulation of MMPs with naringenin treatment was putatively mediated by the NF-kB pathway. However, further studies are required to identify the mechanism by which naringenin causes inhibition of NF-KB pathway. In conclusion, naringenin can be a potential natural therapeutic agent for the treatment of osteoarthritis. Therefore, clinical studies on the effect of naringenin on human osteoarthritis should be planned.

\section{Acknowledgments}

The authors are grateful to the academic staff of Dalian University for their help and support throughout the course of this study. This work was supported by an in-house grant of the Dalian University.

7. Garnero P, Rousseau JC, Delmas PD. Molecular basis and clinical use of biochemical markers of bone, cartilage, and synovium in joint diseases. Arthritis Rheum 2000; 43: 953-968, doi: 10.1002/1529-0131(200005)43:5<953::AIDANR1 > 3.0.CO;2-Q.

8. Lin PM, Chen CT, Torzilli PA. Increased stromelysin-1 (MMP-3), proteoglycan degradation (3B3- and 7D4) and collagen damage in cyclically load-injured articular cartilage. Osteoarthritis Cartilage 2004. 12: 485-496, doi: 10.1016/ j.joca.2004.02.012.

9. Ameye LG, Chee WS. Osteoarthritis and nutrition. From nutraceuticals to functional foods: a systematic review of the scientific evidence. Arthritis Res Ther 2006; 8: R127, doi: 10.1186/ar2016.

10. Wolfe F, Zhao S, Lane N. Preference for nonsteroidal antiinflammatory drugs over acetaminophen by rheumatic disease patients: a survey of 1,799 patients with osteoarthritis, rheumatoid arthritis, and fibromyalgia. Arthritis Rheum 2000. 43: 378-385.

11. Lanas A, Hunt R. Prevention of anti-inflammatory druginduced gastrointestinal damage: benefits and risks of therapeutic strategies. Ann Med 2006; 38: 415-428, doi: 10.1080/ 07853890600925843. 
12. Henry D, Lim LL, Garcia Rodriguez LA, Perez Gutthann S, Carson JL, Griffin M, et al. Variability in risk of gastrointestinal complications with individual non-steroidal anti-inflammatory drugs: results of a collaborative meta-analysis. BMJ 1996; 312: 1563-1566, doi: 10.1136/bmj.312.7046.1563.

13. Jeong JH, Moon SJ, Jhun JY, Yang EJ, Cho ML, Min JK. Eupatilin exerts antinociceptive and chondroprotective properties in a rat model of osteoarthritis by downregulating oxidative damage and catabolic activity in chondrocytes. PLoS One 2015; 10: e0130882, doi: 10.1371/journal.pone. 0130882.

14. Park JS, Kim DK, Shin HD, Lee HJ, Jo HS, Jeong JH, et al. Apigenin regulates interleukin-1beta-induced production of matrix metalloproteinase both in the knee joint of rat and in primary cultured articular chondrocytes. Biomol Ther 2016; 24: 163-170, doi: 10.4062/biomolther.2015.217.

15. Akhtar N, Haqqi TM. Current nutraceuticals in the management of osteoarthritis: a review. Ther Adv Musculoskelet Dis 2012; 4: 181-207, doi: 10.1177/1759720X11436238.

16. Vallverdu-Queralt A, Odriozola-Serrano I, Oms-Oliu G, Lamuela-Raventos RM, Elez-Martinez P, Martin-Belloso O. Changes in the polyphenol profile of tomato juices processed by pulsed electric fields. J Agric Food Chem 2012; 60: 9667-9672, doi: 10.1021/jf302791k.

17. Al-Rejaie SS, Abuohashish HM, Al-Enazi MM, Al-Assaf AH, Parmar MY, Ahmed MM. Protective effect of naringenin on acetic acid-induced ulcerative colitis in rats. World J Gastroenterol 2013; 19: 5633-5644, doi: 10.3748/wjg.v19.i34.5633.

18. Martinez RM, Pinho-Ribeiro FA, Steffen VS, Caviglione CV, Vignoli JA, Barbosa DS, et al. Naringenin Inhibits UVB Irradiation-Induced Inflammation and oxidative stress in the skin of hairless mice. J Nat Prod 2015; 78: 1647-1655, doi: 10.1021/acs.jnatprod.5b00198.

19. Chtourou $Y$, Fetoui H, Jemai R, Ben Slima A, Makni M, Gdoura R. Naringenin reduces cholesterol-induced hepatic inflammation in rats by modulating matrix metalloproteinases-2, 9 via inhibition of nuclear factor kappaB pathway. Eur J Pharmacol 2015; 746: 96-105, doi: 10.1016/j.ejphar. 2014.10.027.

20. Skehan P, Storeng R, Scudiero D, Monks A, McMahon J, Vistica $D$, et al. New colorimetric cytotoxicity assay for anticancer-drug screening. J Natl Cancer Inst 1990; 82: 1107-1112, doi: 10.1093/jnci/82.13.1107.

21. Ye J, Coulouris G, Zaretskaya I, Cutcutache I, Rozen S, Madden TL. Primer-BLAST: a tool to design target-specific primers for polymerase chain reaction. BMC Bioinformatics 2012; 13: 134, doi: 10.1186/1471-2105-13-134.

22. Abramson SB, Attur M, Yazici Y. Prospects for disease modification in osteoarthritis. Nat Clin Pract Rheumatol 2006; 2: 304-312, doi: 10.1038/ncprheum0193.

23. Goldring MB, Birkhead JR, Suen LF, Yamin R, Mizuno S, Glowacki J, et al. Interleukin-1 beta-modulated gene expression in immortalized human chondrocytes. J Clin Invest 1994; 94: 2307-2316, doi: 10.1172/JCl117595.

24. Mengshol JA, Vincenti MP, Coon $\mathrm{Cl}$, Barchowsky A, Brinckerhoff CE. Interleukin-1 induction of collagenase 3 (matrix metalloproteinase 13) gene expression in chondrocytes requires p38, c-Jun $\mathrm{N}$-terminal kinase, and nuclear factor kappaB: differential regulation of collagenase 1 and collagenase 3. Arthritis Rheum 2000; 43: 801-811, doi: 10.1002/15290131(200004)43:4 < 801::AID-ANR10 > 3.0.CO;2-4.
25. Koshy PJ, Lundy CJ, Rowan AD, Porter S, Edwards DR, Hogan $A$, et al. The modulation of matrix metalloproteinase and ADAM gene expression in human chondrocytes by interleukin-1 and oncostatin $\mathrm{M}$ : a time-course study using real-time quantitative reverse transcription-polymerase chain reaction. Arthritis Rheum 2002; 46: 961-967, doi: 10.1002/art.10212.

26. Mach DB, Rogers SD, Sabino MC, Luger NM, Schwei MJ, Pomonis JD, et al. Origins of skeletal pain: sensory and sympathetic innervation of the mouse femur. Neuroscience 2002, 113: 155-166.

27. Fernihough J, Gentry C, Malcangio M, Fox A, Rediske J, Pellas $\mathrm{T}$, et al. Pain related behavior in two models of osteoarthritis in the rat knee. Pain 2004; 112: 83-93, doi: 10.1016/j.pain.2004.08.004.

28. Felson DT, Chaisson CE, Hill CL, Totterman SM, Gale ME, Skinner KM, et al. The association of bone marrow lesions with pain in knee osteoarthritis. Ann Intern Med 2001; 134: 541-549, doi: 10.7326/0003-4819-134-7-20010403000007.

29. Hill CL, Gale DG, Chaisson CE, Skinner K, Kazis L, Gale $M E$, et al. Knee effusions, popliteal cysts, and synovial thickening: association with knee pain in osteoarthritis. $J$ Rheumatol 2001; 28: 1330-1337.

30. Wluka AE, Wolfe R, Stuckey S, Cicuttini FM., How does tibial cartilage volume relate to symptoms in subjects with knee osteoarthritis? Ann Rheum Dis 2004; 63: 264-268, doi: 10.1136/ard/2003.007666.

31. Freemont AJ, Hampson V, Tilman R, Goupille P, Taiwo $Y$, Hoyland JA. Gene expression of matrix metalloproteinases 1,3 , and 9 by chondrocytes in osteoarthritic human knee articular cartilage is zone and grade specific. Ann Rheum Dis 1997; 56: 542-549, doi: 10.1136/ard.56.9.542.

32. Yoshihara $\mathrm{Y}$, Nakamura H, Obata K, Yamada H, Hayakawa $\mathrm{T}$, Fujikawa $\mathrm{K}$, et al. Matrix metalloproteinases and tissue inhibitors of metalloproteinases in synovial fluids from patients with rheumatoid arthritis or osteoarthritis. Ann Rheum Dis 2000; 59: 455-461, doi: 10.1136/ard.59.6.455.

33. Little CB, Barai A, Burkhardt D, Smith SM, Fosang AJ, Werb Z, et al. Matrix metalloproteinase 13-deficient mice are resistant to osteoarthritic cartilage erosion but not chondrocyte hypertrophy or osteophyte development. Arthritis Rheum 2009; 60: 3723-3733, doi: 10.1002/art.25002.

34. Echtermeyer F, Bertrand J, Dreier R, Meinecke I, Neugebauer K, Fuerst M, et al. Syndecan-4 regulates ADAMTS-5 activation and cartilage breakdown in osteoarthritis. Nat Med 2009; 15: 1072-1076, doi: 10.1038/nm.1998.

35. Stanton H, Rogerson FM, East CJ, Golub SB, Lawlor KE, Meeker CT, et al. ADAMTS5 is the major aggrecanase in mouse cartilage in vivo and in vitro. Nature 2005; 434: 648-652, doi: 10.1038/nature03417.

36. Handel ML, McMorrow LB, Gravallese EM. Nuclear factorkappa $B$ in rheumatoid synovium. Localization of p50 and p65. Arthritis Rheum 1995; 38: 1762-1770, doi: 10.1002/ art.1780381209.

37. Roman-Blas JA, Jimenez SA. NF-kappaB as a potential therapeutic target in osteoarthritis and rheumatoid arthritis. Osteoarthritis Cartilage 2006; 14: 839-848, doi: 10.1016/j. joca.2006.04.008.

38. Shi Y, Dai J, Liu H, Li RR, Sun PL, Du Q, et al. Naringenin inhibits allergen-induced airway inflammation and airway 
responsiveness and inhibits NF-kappaB activity in a murine model of asthma. Can J Physiol Pharmacol 2009; 87: 729-735, doi: 10.1139/Y09-065.

39. Hamalainen $M$, Nieminen $R$, Vuorela $P$, Heinonen $M$, Moilanen E. Anti-inflammatory effects of flavonoids: genistein, kaempferol, quercetin, and daidzein inhibit STAT-1 and NF-kappaB activations, whereas flavone, isorhamnetin, naringenin, and pelargonidin inhibit only NF-kappaB activation along with their inhibitory effect on iNOS expression and NO production in activated macrophages. Mediators Inflamm 2007; 2007: 45673, doi: 10.1155/2007/ 45673. 